\title{
THE DETERMINATION OF THE MORPHOLOGICAL TYPES OF GALAXY CLUSTERS USING CLUSTER CARTOGRAPHY
}

\author{
E.A. Panko, S.I. Emelyanov \\ V.O. Suckomlinsky Nikolaev National University, Kalinenkov Astronomical Observatory \\ Nikolskaya, 24, Nikolaev, 54030, Ukraine \\ panko.elena@gmail.com
}

\begin{abstract}
We discuss the possibility of using the Cluster Cartography set for the determined the morphological types of galaxy clusters. The applied morphological scheme was proposed by Panko (2013). The morphological types are determined using numerical criteria based on three parameters: concentration to the cluster center, the signs of preferential direction or plane in the cluster (filamentary substructure), and the positions of the brightest galaxies. However, structures like galaxy clusters need visual preview for classification. The Cluster Cartography set constructs the individual cluster map in different forms and allows to estimate previously cluster type.
\end{abstract}

Keywords: Galaxies: clusters: morphological types.

\section{Introduction}

The morphology of galaxy clusters reflects the physical conditions in the cluster space. One can note the galaxy clusters have a special place in hierarchy of large-scale structures. They are the part of a continuous range of large-scale construction of Universe: galaxies $\Rightarrow$ groups $\Rightarrow$ clusters $\Rightarrow$ superclusters $\Rightarrow$ cosmological large scale structure. Galaxy cluster virialization time is about $10^{9} \mathrm{yr}$, and it is less then Hubble time. In contrary, galaxy superclusters virialization time - about $10^{10.5}$ - is bigger then Hubble time. As a result the galaxy clusters are not biggest bound structures in the Universe, they are only biggest virialized ones. The galaxy clusters are small in comparison to Universe. At the same time, on the galaxy clusters scale, their components have not had a chance to separate during collapse and a cluster is probably a representative sample of the Universe. In particulary, the part of dark matter (DM) in galaxy clusters must be the same, as in whole Universe. The determination of morphological types of galaxy clusters will be useful for detailed study of the large scale structures.

Panko (2013) summarized the classical schemes, including both famous Bautz - Morgan (1970), Rood - Sastry (1971) systems and less popular López-Cruz at al. (1997) and López-Cruz \& Gaztanaga (2001) ones. Improved and integrated scheme (Panko 2013) allows to assign the morphological types corresponding to cluster "concentration" (from $C$ - compact, to $I$ - intermediate, and $O$ - open), "flatness signs" ( $L-$ line or $F$ - flat, and no symbol if no indication of flatness is present) and the role of bright galaxies (cD or $B G$, if the bright cluster members role is significant). Other peculiarities are noted as $P$. "Flatness signs" can correspond to filamentary substructure or preferential plane in cluster. The designations can be combined, for example $C F \mathrm{c} D$ or $I L P$. Unfortunately, like to morphology of galaxies case, programmatic way does not allow to distinguish morphology without visual preview and control.

We create the Cluster Cartography set (hereafter $\mathrm{CC}$ ) for simplification of the galaxy clusters classification.

\section{Observational Data}

The CC set was create for morphology classification of galaxy clusters of the "A Catalogue of Galaxy Clusters and Groups" (Panko \& Flin, 2006, hereafter PF). The PF Catalogue was constructed on Münster Red Sky Survey Galaxy Catalogue (Ungrue et al., 2003, hereafter MRSS) as the observational basis. Each PF galaxy cluster has the list of galaxies in the cluster field inclusive for each galaxy information accordingly to MRSS, specifically $R A_{2000}$ and $D e c_{2000}, r_{F}$ magnitude, major and minor axes and positional angle of major axis of galaxy best-fitted ellipse (Ungrue et al., 2003).

\section{The Cluster Cartography set}

The cluster map is constructed in rectangular coordinates recalculated into arcseconds. The equatorial coordinates were recalculated to rectangular ones centered in the cluster center by usual way. The CC set allows to construct the cluster map in four modes: 

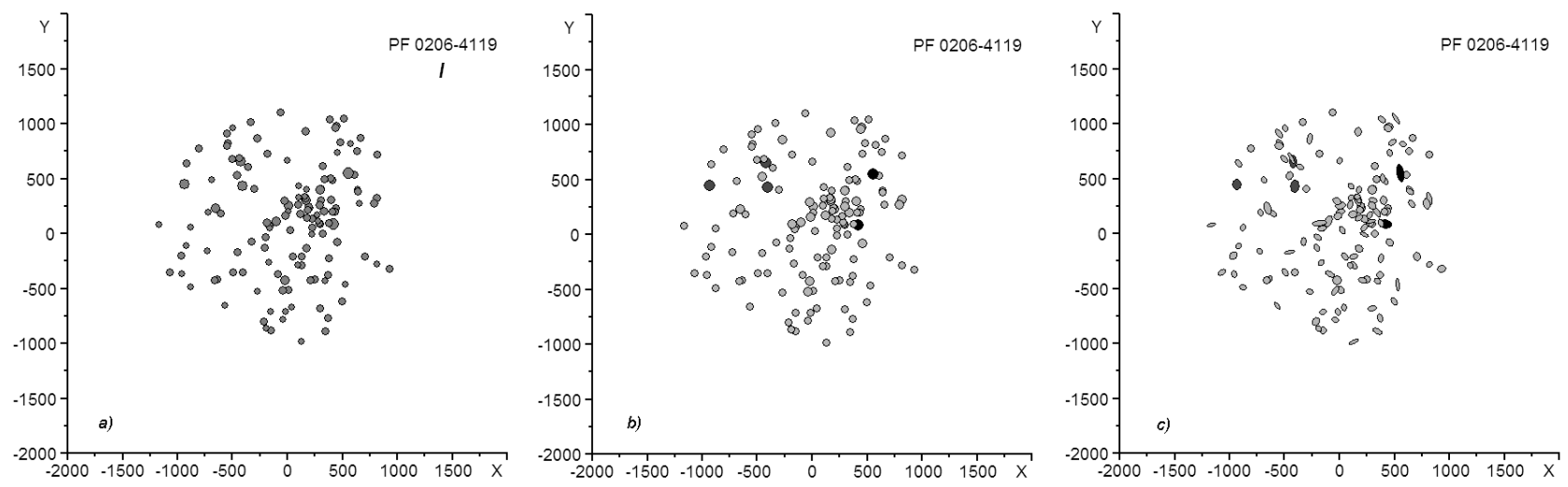

Figure 1: The modes of cluster mapping. All maps constructed for the same I-type cluster PF 0206-4119.

- all symbols are circles and have the same size;

- all symbols are circles and the symbol size corresponds to magnitude of galaxy (Fig. $1 a$ and $b$ );

- the symbol size corresponds to magnitude of galaxy and each symbol illustrates the galaxy shape and orientation in the projection on the celestial sphere (Fig. 1c);

- the symbols illustrates the galaxy shape and orientation in the projection on the celestial sphere, but size of symbol corresponds to galaxy size in arcseconds.

Additionally brightest galaxies can be marked by darker gray shades (Fig. $1 b$ and $c$ ).

The shown in Fig. $1 b$ CC mode allows to estimate the morphological type according to Panko (2013) and the select the clusters for numerical definition. The analyze of distribution of ellipticities of galaxies in the cluster notes to one more parameter in classification - the part of E-type galaxies (Panko \& Flin, 2014). E-rich and S-rich galaxy clusters were recognized by Oemler in 1974. The galaxy shape and orientation mode was added for previous estimation of positions of E-type galaxies in the cluster field. In our data set (accordingly to MRSS) we can divide galaxies only to elliptical and non-elliptical. Galaxies with ellipticity $E>0.5$ can be lenticular or spiral (near edge-on view) or interactive ones. Positions of these galaxies in E-poor clusters is important in connection with Morphology Density relation (Dressler, 1980).

The diameter of circle symbol $m^{\prime}$ was calculated using galaxy magnitude $m$ as:

$$
m^{\prime}=3 \cdot 2^{0.6(18.5-m)}+6
$$

The coefficients can be changed in case of need, but for typical map size $-4000 \times 4000$ arcsec - the symbol sizes calculated according to (1) are optimal.

The sizes of major and minor axes $(2 a$ and $2 b)$ for shown in Fig. $1 c$ CC mode are calculated from $m^{\prime}$ and ellipticity $E$ as:

$$
2 a=\frac{m^{\prime}}{\sqrt[4]{1-2 E+E^{2}}} ; \quad 2 b=\frac{\left(m^{\prime}\right)^{2}}{2 a}
$$

Note, the ellipticity $E=1-\frac{b}{a}$. The equations (2) transform the circle to ellipse with the same area and connect symbol axes with galaxy magnitude. We use MRSS data for maps shown real galaxy size with magnitudes noted as shades of gray in CC mode 4.

\section{Conclusion}

We tested the CC set in different modes on 247 rich $\mathrm{PF}$ galaxy clusters. It allows to estimate galaxy cluster morphological type quickly. We will use the $\mathrm{CC}$ set in future work for study of $1746 \mathrm{PF}$ galaxy clusters with richness 50 and more. About of 1200 galaxy clusters in this list have no morphological types and using the CC set allows to improve our data set.

Acknowledgements. This research has made use of NASA's Astrophysics Data System.

\section{References}

Bautz P. \& Morgan W.W.: 1970, ApJ, 162, L149.

Dressler A.: 1980 ApJ, 236, 351.

López-Cruz O., Yee H.K.C., Brown J.P., et al.: 1997, ApJ, 475, L97.

López-Cruz O. \& Gaztanaga E.: 2001, arXiv:astroph/0009028.

Oemler A.Jr.: 1974, ApJ, 194, 1.

Panko E. \& Flin P.: 2006, J. Astr. Data, 12, 1.

Panko E.: 2013, Odessa Astr. Publ., 26, 90.

Panko E. \& Flin P.: 2014, Odessa Astr. Publ., 27, 32.

Rood H.J. \& Sastry G.N.: 1971, PASP, 83, 313.

Ungrue R., Seitter W.C. \& Duerbeck, H.W.: 2003, J. Astr. Data, 9,1. 